\title{
Synthesis of heteroglycoclusters by using orthogonal chemoselective ligations
}

\author{
Baptiste Thomas ${ }^{1}$, Michele Fiore ${ }^{1}$, Isabelle Bossu ${ }^{1}$, Pascal Dumy ${ }^{1}$ \\ and Olivier Renaudet ${ }^{*} 1,2$
}

\section{Letter}

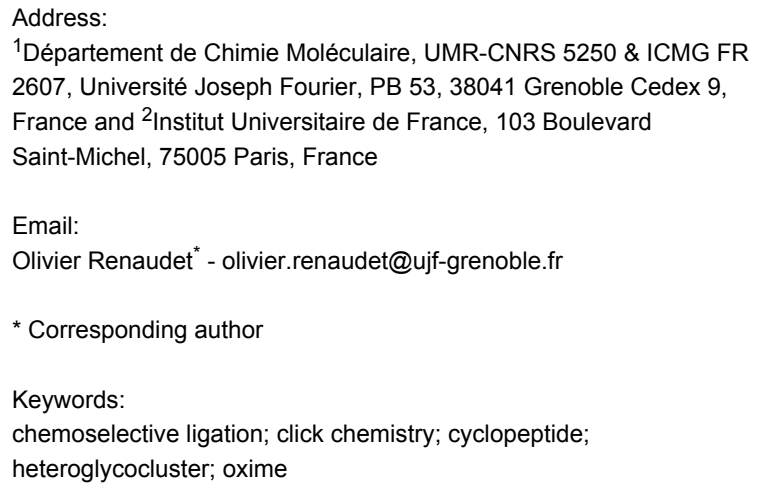

${ }^{1}$ Département de Chimie Moléculaire, UMR-CNRS 5250 \& ICMG FR 2607, Université Joseph Fourier, PB 53, 38041 Grenoble Cedex 9, France and ${ }^{2}$ Institut Universitaire de France, 103 Boulevard Saint-Michel, 75005 Paris, France

Beilstein J. Org. Chem. 2012, 8, 421-427. doi:10.3762/bjoc. 8.47

Received: 20 January 2012

Accepted: 29 February 2012

Published: 20 March 2012

This article is part of the Thematic Series "Synthesis in the glycosciences II".

Guest Editor: T. K. Lindhorst

(C) 2012 Thomas et al; licensee Beilstein-Institut. License and terms: see end of document.

\begin{abstract}
Synthetic heteroglycoclusters are being subjected to increasing interest due to their potential to serve as selective ligands for carbohydrate-binding proteins. In this paper, we describe an expedient strategy to prepare cyclopeptides displaying well-defined distributions and combinations of carbohydrates. By using both oxime ligation and copper(I)-catalyzed alkyne-azide cycloaddition, two series of compounds bearing binary combinations of $\alpha \mathrm{Man}, \alpha \mathrm{Fuc}$ or $\beta \mathrm{Lac}$ in an overall tetravalent presentation, and either 2:2 or 3:1 relative proportions, have been prepared.
\end{abstract}

\section{Introduction}

Multivalent interactions between carbohydrates and proteins play key roles in diverse biological events, including fertilization, cell-cell communication, host-pathogen interactions, immune response and cancer metastasis [1]. Synthetic molecules displaying multiple copies of a sugar binding motif, called (homo)glycoclusters, represent attractive tools for studying these complex recognition processes as well as for developing biological applications, for example, the inhibition of infections by pathogens such as viruses or bacteria [2-5]. In a suitable density and spatial display, clusters of carbohydrate indeed allow multiple contacts with a target protein, thus increasing avidity by means of the "glycoside cluster effect" $[6,7]$. While the recent progress in glycomics has led to the design of glycoclusters active at nanomolar concentration [8-10], the achievement of selective binding remains challenging because of the close structural similarities of the binding sites of proteins specific for the same carbohydrate moiety. 
Interestingly, recent reports have highlighted that the association of different sugar units instead of a single motif, namely heteroglycocluster, reflects the presence of sugars found in native biological systems more closely than homoglycocluster does. Although recognition mechanisms are not fully understood, these studies suggest that heteroglycoclusters should interact with proteins through distinct binding sites, which may influence both affinity and selectivity [11-25]. In this context, we previously reported a combinatorial procedure to prepare libraries of heteroglycoclusters displaying sugars and/or amino acids at randomized positions on a topological cyclopeptide scaffold [26]. Deconvolution of the resulting libraries by affinity chromatography allowed the selection of heteroglycoclusters that were proved to be useful for exploring the surrounding regions of the binding pocket in a model lectin. Although it is easy to handle, this combinatorial procedure leads to the formation of inseparable mixtures of regioisomers, which precludes their utilization for further assays with relevant biological targets. In order to circumvent this drawback, we herein report the synthesis of similar heteroglycoclusters by using a protocol based on orthogonal chemoselective ligations. Two series of compounds containing different combinations of two different sugars have been designed (Figure 1). In one series (heteroglycocluster 2:2), two $\alpha$ Man, $\alpha$ Fuc and/or $\beta$ Lac are conjugated at alternate positions into the tetravalent cyclopeptide sequence, whereas the second series (heteroglycocluster $3: 1)$ contains one of a given sugar and three of another.

\section{Results and Discussion}

Glycoclusters are classically constructed from a molecular scaffold containing multiple anchoring sites that can be functional- ized with sugars by using a single coupling reaction. By contrast, the chemical access of heteroglycoclusters is not trivial because it requires the controlled conjugation of different sugars at a precise position into the scaffold to obtain a well-defined distribution. Taking advantage of our experience in bioconjugation methods, we decided to explore two chemoselective strategies to achieve this purpose. We first selected the oxime ligation since we have previously used this approach successfully for the preparation of sophisticated molecular systems, such as synthetic vaccines [27,28], immunomodulators [29], lectin ligands [30] or vectors of hepatocytes [31]. As the second strategy, we have chosen the well-known copper(I)-catalyzed alkyne-azide cycloaddition (CuAAC) [32,33], which is intensively exploited for the conjugation of sugars to both molecular and biological systems [34,35]. Besides being fully compatible with carbohydrate and peptide chemistries, oximation and CuAAC reactions offer the advantage of being orthogonal $[36,37]$, therefore allowing a controlled assembly process with a minimized risk of side reactions. The 2:2 series of heteroglycoclusters was prepared from the aminooxy [38-40] and propargyl [41] glycosides 1-3 (Figure 1) and the cyclopeptide 4 (Scheme 1). This scaffold containing two lysines (Lys) functionalized with an aldehyde and two norleucines (Nle) bearing an azide group was prepared by using a strategy adapted from the procedure described previously [42].

In the first step, two copies of aminooxy $\alpha$ Man 1a, $\alpha$ Fuc 2a and $\beta$ Lac 3a were coupled to 4 , affording divalent oxime-linked compounds 5-Man, 5-Fuc, and 5-Lac, respectively. The oxime ligation was performed at $37^{\circ} \mathrm{C}$ in aqueous acidic buffer with 2 equiv of sugars per anchoring site. After $3 \mathrm{~h}$, complete reac- a)

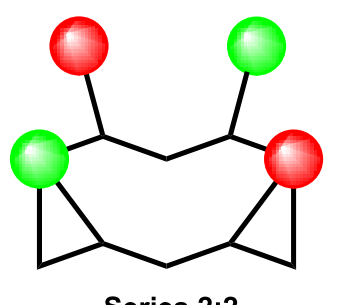

b)

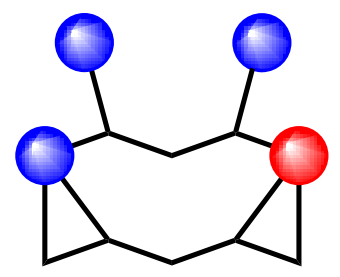

Series $3: 1$ c) $=\alpha-D-M a n$

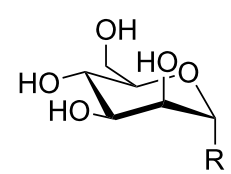

1a: $\mathrm{R}=-\mathrm{ONH}_{2}$

1b: $\mathrm{R}=-\mathrm{OCH}_{2} \mathrm{C} \equiv \mathrm{CH}$ $=\alpha-$ L-Fuc

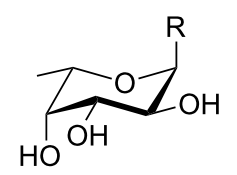

2a: $\mathrm{R}=-\mathrm{ONH}_{2}$

2b: $\mathrm{R}=-\mathrm{OCH}_{2} \mathrm{C} \equiv \mathrm{CH}$

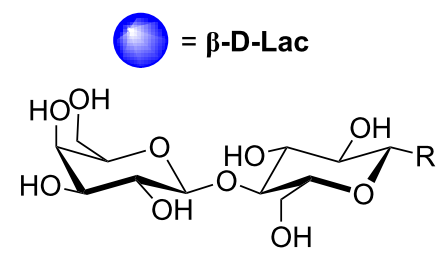

3a: $\mathrm{R}=-\mathrm{ONH}_{2}$

3b: $\mathrm{R}=-\mathrm{OCH}_{2} \mathrm{C} \equiv \mathrm{CH}$

Figure 1: (a) Schematic representation of a heteroglycocluster of the 2:2 series containing Man and Fuc. (b) Schematic representation of a heteroglycocluster of the 3:1 series containing Lac and Man. (c) Structure of carbohydrates used for the construction of heteroglycoclusters. 


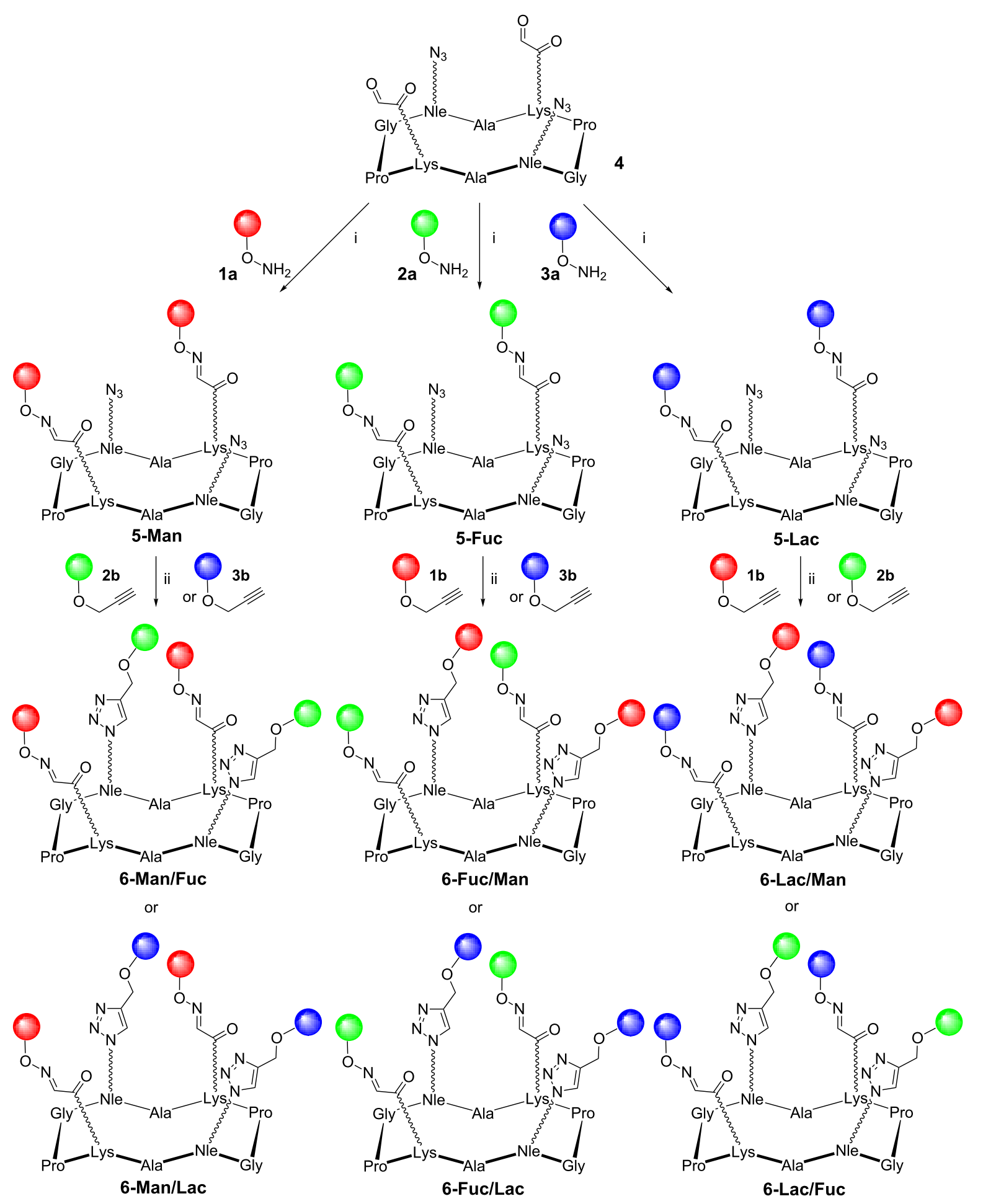

Scheme 1: Synthesis of heteroglycoclusters of the 2:2 series. Reagents and conditions: (i) $0.1 \% \mathrm{TFA}$ in $\mathrm{H}_{2} \mathrm{O}$; (ii) $\mathrm{Cu}$ micropowder, $t$ - $\mathrm{BuOH}$, $\mathrm{AcONH}$ $100 \mathrm{mM} \mathrm{pH} 7.4(1: 1, v / v)$. The wavy bond represents the aliphatic part (i.e., $\left.\left(\mathrm{CH}_{2}\right)_{4}\right)$ of the lysine (Lys) and the norleucine $(\mathrm{Nle})$ side chain.

tions were observed by analytical HPLC. The excess of sugar was then quenched by the addition of acetone, and the resulting crude mixtures were used for $\mathrm{CuAAC}$ without further purification. The efficiency of CuAAC clearly depends on the experi- mental conditions $[34,35]$. The choice of the solvent and of the copper(I) catalyst (delivered either using CuI, copper micropowder, or $\mathrm{CuSO}_{4}$ and sodium ascorbate as reducing agent) and the utilization of microwave or ultrasonic irradiation are para- 
meters that can influence the reaction kinetics, improve the yields and sometimes prevent side reactions. In a previous study, we observed that a tetravalent glycocluster can be obtained in good yields and as a unique 1,4-regioisomer by using a catalytic amount of copper micropowder in a mixture of isopropanol and ammonium acetate buffer [42]. Therefore we decided to follow similar conditions in this study with propargyl glycosides $\alpha$ Man 1b, $\alpha$ Fuc $2 \mathbf{b}$ and $\beta$ Lac 3b. Here again, each reaction occurred quantitatively, as shown in RP-HPLC profiles of the crude reaction mixtures (see Supporting Information File 1). After removal of solid copper by filtration and semipreparative HPLC, six tetravalent heteroglycoclusters combining two sugars (e.g., 6-Man/Fuc, 6-Man/Lac, 6-Fuc/ Man, 6-Fuc/Lac, 6-Lac/Man and 6-Lac/Fuc) were obtained in excellent conversion rate and purity and unambiguously characterized by mass spectrometry (Table 1).

To demonstrate the reliability of our protocol, a new series of 3:1 heteroglycoclusters was prepared from the same carbohydrate building blocks and the cyclopeptide 7. Similar experimental conditions were followed, with the exception of the stoichiometry of reagents (see Experimental section). In this series, one oxime linkage was formed from 7 by using aminooxy $\alpha$ Man 1a, $\alpha$ Fuc 2a and $\beta$ Lac 3a, and three CuAAC reactions were subsequently performed with propargyl glycosides $\alpha$ Man $\mathbf{1 b}, \alpha$ Fuc $\mathbf{2 b}$ and $\beta$ Lac $\mathbf{3 b}$ as described above (Scheme 2).

No difference in reactivity from the previous $2: 2$ series was observed, thereby confirming the efficiency of this strategy for the preparation of well-defined heteroglycoclusters.

\section{Conclusion}

In this paper we have described an expedient and controlled assembly protocol to prepare heteroglycoclusters similar to those obtained previously from randomized combinatorial libraries [26]. Following two orthogonal chemoselective reactions, cyclopeptides 4 and 7 were successively reacted with aminooxy glycosyls 1-3a and propargyl glycosides 1-3b. Twelve novel oxime and triazole linked-heteroglycoclusters displaying well-defined distributions and combinations of carbohydrates were thus obtained in excellent yields and purity.

\section{Experimental \\ Standard procedures for the heteroglyco- cluster assembly}

\section{Series $2: 2$}

A solution of $4(4.0 \mathrm{mg}, 3.4 \mu \mathrm{mol})$ and $\mathbf{2 a}(2.6 \mathrm{mg}, 14.2 \mu \mathrm{mol}$, 4 equiv) was stirred at $37{ }^{\circ} \mathrm{C}$ in $0.1 \%$ TFA in $\mathrm{H}_{2} \mathrm{O}(400 \mu \mathrm{L})$. After $3 \mathrm{~h}$, analytical HPLC revealed the total conversion of $4 \mathrm{a}$ into 5-Fuc. Analytical HPLC: $t_{\mathrm{R}} 9.34 \mathrm{~min}$ (gradient: 5 to $100 \%$ $\mathrm{B}$ in $20 \mathrm{~min}) ; \mathrm{ESI}^{+}-\mathrm{MS}(\mathrm{m} / z):[\mathrm{M}+\mathrm{Na}]^{+}$calcd for $\mathrm{C}_{60} \mathrm{H}_{96} \mathrm{~N}_{20} \mathrm{O}_{22} \mathrm{Na}$, 1471.7; found, 1471.8. After the addition of acetone $(100 \mu \mathrm{L})$ to the crude, the reaction mixture was lyophilized, then resuspended with $t-\mathrm{BuOH} / \mathrm{AcONH}_{4} 100 \mathrm{mM}$ pH $7.4(500 \mu \mathrm{L}, 1: 1 v / v)$. Compound $1 \mathbf{b}(4.0 \mathrm{mg}, 10.5 \mu \mathrm{mol}$, 3 equiv) and copper micropowder $(455 \mu \mathrm{g}, 7.0 \mu \mathrm{mol})$ were next added to the solution and the resulting mixture was left under stirring at room temperature. After $4 \mathrm{~h}$, copper was removed by centrifugation and the supernatant purified by semipreparative HPLC. Compound 6-Fuc/Man was obtained in $91 \%$ yield

Table 1: Outcome of the orthogonal ligation procedure.

\begin{tabular}{|c|c|c|c|c|}
\hline compound & yield $^{a}$ & MS calcd ${ }^{b}$ & MS found ${ }^{c}$ & $t_{\mathrm{R}}(\min )^{\mathrm{d}}$ \\
\hline 6-Man/Fuc & $83 \%$ & $1885.9\left(\mathrm{C}_{78} \mathrm{H}_{124} \mathrm{~N}_{20} \mathrm{O}_{34}\right)$ & 1886.0 & 7.79 \\
\hline 6-Man/Lac & $99 \%$ & $2242.9\left(\mathrm{C}_{90} \mathrm{H}_{145} \mathrm{~N}_{20} \mathrm{O}_{46}\right)$ & 2242.3 & 7.43 \\
\hline 6-Fuc/Man & $98 \%$ & $1885.9\left(\mathrm{C}_{78} \mathrm{H}_{124} \mathrm{~N}_{20} \mathrm{O}_{34}\right)$ & 1886.0 & 7.73 \\
\hline 6-Fuc/Lac & $98 \%$ & $2210.0\left(\mathrm{C}_{90} \mathrm{H}_{145} \mathrm{~N}_{20} \mathrm{O}_{44}\right)$ & 2210.3 & 7.62 \\
\hline 6-Lac/Man & $94 \%$ & $2242.9\left(\mathrm{C}_{90} \mathrm{H}_{145} \mathrm{~N}_{20} \mathrm{O}_{46}\right)$ & 2242.2 & 7.31 \\
\hline 6-Lac/Fuc & $93 \%$ & $2210.0\left(\mathrm{C}_{90} \mathrm{H}_{145} \mathrm{~N}_{20} \mathrm{O}_{44}\right)$ & 2210.3 & 7.60 \\
\hline 8-Man/Fuc & $85 \%$ & $1880.9\left(\mathrm{C}_{79} \mathrm{H}_{126} \mathrm{~N}_{21} \mathrm{O}_{32}\right)$ & 1881.1 & 7.88 \\
\hline 8-Man/Lac & $87 \%$ & $2415.0\left(\mathrm{C}_{97} \mathrm{H}_{156} \mathrm{~N}_{21} \mathrm{O}_{50}\right)$ & 2415.4 & 7.30 \\
\hline 8-Fuc/Man & $91 \%$ & $1912.9\left(\mathrm{C}_{79} \mathrm{H}_{126} \mathrm{~N}_{21} \mathrm{O}_{34}\right)$ & 1913.2 & 7.66 \\
\hline 8-Fuc/Lac & $90 \%$ & $2400.0\left(\mathrm{C}_{97} \mathrm{H}_{156} \mathrm{~N}_{21} \mathrm{O}_{49}\right)$ & 2399.3 & 7.46 \\
\hline 8-Lac/Man & $89 \%$ & $2092.0\left(\mathrm{C}_{85} \mathrm{H}_{136} \mathrm{~N}_{21} \mathrm{O}_{40}\right)$ & 2091.2 & 7.46 \\
\hline 8-Lac/Fuc & $91 \%$ & $2043.9\left(\mathrm{C}_{85} \mathrm{H}_{136} \mathrm{~N}_{21} \mathrm{O}_{37}\right)$ & 2043.1 & 7.83 \\
\hline
\end{tabular}

aYields were calculated by integrating the peak corresponding to the expected compound in the crude HPLC profile. Isolated yields are given in the Experimental section. ${ }^{b}$ Calculated mass for $[\mathrm{M}+\mathrm{H}]^{+}$. ${ }^{\mathrm{C}} \mathrm{MS}$ analysis was performed by electrospray ionization method in positive mode. ${ }^{\mathrm{d} R P}$-HPLC retention time using a linear gradient $A / B, 95: 5$ to $0: 100$ in $20 \mathrm{~min}$, flow: $1.0 \mathrm{~mL} / \mathrm{min}, \lambda=214 \mathrm{~nm}$ and $250 \mathrm{~nm}$ (column: nucleosil $300-5 \mathrm{C}_{18}$; solvent $A$ : $0.09 \%$ TFA in $\mathrm{H}_{2} \mathrm{O}$, solvent $\mathrm{B}$ : $0.09 \%$ TFA in $90 \%$ acetonitrile). 


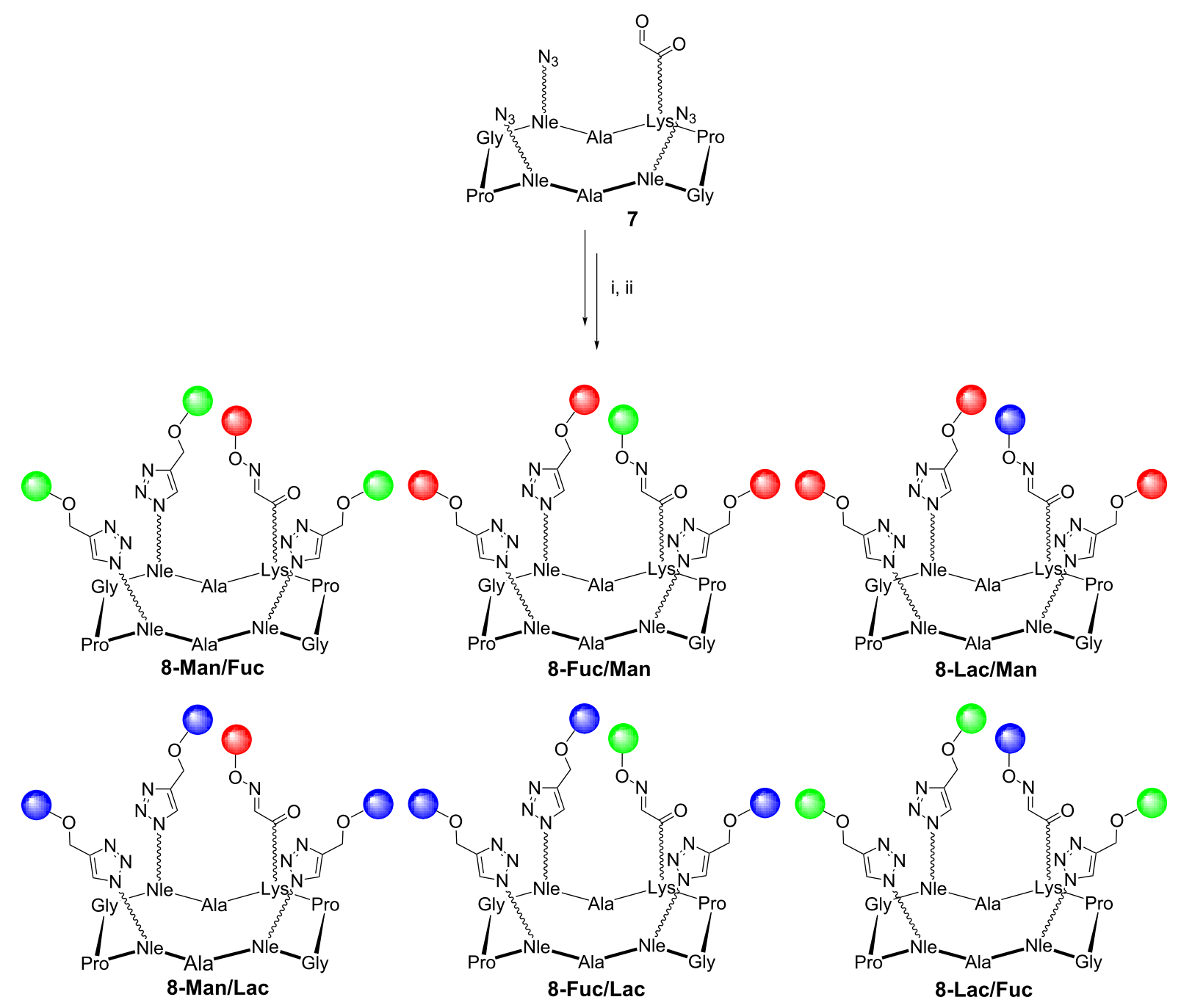

Scheme 2: Synthesis of heteroglycoclusters of the $3: 1$ series. Reagents and conditions: (i) $\mathbf{1 a}, \mathbf{2 a}$ or $\mathbf{3 a}, \mathbf{0 . 1} \%$ TFA in $\mathrm{H}_{2} \mathrm{O}$; (ii) $\mathbf{1 b}, \mathbf{2 b}$ or $\mathbf{3 b}$, Cu micropowder, $t$-BuOH, $\mathrm{AcONH}_{4} 100 \mathrm{mM} \mathrm{pH} 7.4(1: 1, \mathrm{v} / \mathrm{v})$.

(5.9 mg). Analytical RP-HPLC: $t_{\mathrm{R}} 7.73 \mathrm{~min}$ (gradient: 5 to $100 \% \mathrm{~B}$ in $20 \mathrm{~min}) ;{ }^{1} \mathrm{H}$ NMR $\left(400 \mathrm{MHz}, \mathrm{D}_{2} \mathrm{O}\right) \delta 8.07(\mathrm{~s}, 1 \mathrm{H}$, $\left.\mathrm{H}_{\text {trz }}\right), 8.06\left(\mathrm{~s}, 1 \mathrm{H}, \mathrm{H}_{\mathrm{trz}}\right), 7.81\left(\mathrm{~s}, 1 \mathrm{H}, \mathrm{H}_{\mathrm{ox}}\right), 7.80\left(\mathrm{~s}, 1 \mathrm{H}, \mathrm{H}_{\mathrm{ox}}\right)$, $5.62\left(\mathrm{~d}, J_{1,2}=4.0 \mathrm{~Hz}, 1 \mathrm{H}, \mathrm{H}-1_{\mathrm{Fuc}}\right), 5.60\left(\mathrm{~d}, J_{1,2}=4.0 \mathrm{~Hz}, 1 \mathrm{H}\right.$, H-1 ${ }_{\text {Fuc }}$ ), 4.99 (bs, 2H, 2H-1 Man $), 4.86-4.68$ (m, 6H, 2H $\mathrm{H}_{\alpha \mathrm{Lys} / \mathrm{Nle}}$, $\left.2 \mathrm{CH}_{2 \text { propargyl }}\right), 4.47-4.35\left(\mathrm{~m}, 10 \mathrm{H}, 2 \mathrm{H}_{\alpha \mathrm{Lys} / \mathrm{Nle}}, 2 \mathrm{CH}_{2 \varepsilon \mathrm{Nle}}\right.$, $\left.2 \mathrm{H}_{\alpha \mathrm{Ala}}, 2 \mathrm{H}_{\alpha \mathrm{Pro}}\right), 4.16-3.63\left(\mathrm{~m}, 28 \mathrm{H}, 2 \mathrm{CH}_{2 \alpha \mathrm{Gly}}, 2 \mathrm{CH}_{2 \delta \text { Pro }}\right.$, $2 \mathrm{H}-2_{\mathrm{Fuc}}, 2 \mathrm{H}-3_{\mathrm{Fuc}}, 2 \mathrm{H}-4_{\mathrm{Fuc}}, 2 \mathrm{H}-5_{\mathrm{Fuc}}, 2 \mathrm{H}-2_{\mathrm{Man}}, 2 \mathrm{H}-3_{\mathrm{Man}}$, $2 \mathrm{H}-4_{\mathrm{Man}}, 2 \mathrm{H}-5_{\mathrm{Man}}, 2 \mathrm{CH}_{2}-6_{\mathrm{Man}}$ ), 3.36-3.20 (m, 4H, 4H $\mathrm{H}_{\varepsilon \mathrm{Lys}}$ ), 2.39-2.29 (m, 2H, 2H $\mathrm{H}_{\beta \mathrm{Pro}}$ ), 2.16-1.27 (m, 36H, 4CH $2 \beta$ Lys/Nle, $\left.4 \mathrm{CH}_{2 \delta \mathrm{Lys} / \mathrm{Nle}}, 4 \mathrm{CH}_{2 \gamma \mathrm{Lys} / \mathrm{Nle}}, 2 \mathrm{H}_{\beta \text { Pro }}, 2 \mathrm{CH}_{2 \gamma \mathrm{Pro}}, 2 \mathrm{CH}_{3 \mathrm{Ala}}\right), 1.22$ $\left(\mathrm{d}, J_{5,6}=6.0 \mathrm{~Hz}, 6 \mathrm{H}, 2 \mathrm{CH}_{3 \mathrm{Fuc}}\right) ; \mathrm{ESI}^{+}-\mathrm{MS}(\mathrm{m} / \mathrm{z}):[\mathrm{M}+\mathrm{H}]^{+}$ calcd for $\mathrm{C}_{78} \mathrm{H}_{124} \mathrm{~N}_{20} \mathrm{O}_{34}, 1885.9$; found, 1886.0. Compounds 6-Man/Fuc, 6-Man/Lac, 6-Fuc/Lac, 6-Lac/Man and 6-Lac/ Fuc were prepared following the same experimental conditions.

\section{Series 3:1}

A solution of $7(6.6 \mathrm{mg}, 5.9 \mu \mathrm{mol})$ and $2 \mathrm{a}(2.2 \mathrm{mg}, 1.2 \mu \mathrm{mol}$, 2 equiv) was stirred at $37{ }^{\circ} \mathrm{C}$ in $0.1 \%$ TFA in $\mathrm{H}_{2} \mathrm{O}(600 \mu \mathrm{L})$. After $3 \mathrm{~h}$, analytical HPLC revealed the total conversion of 7 into the corresponding monovalent intermediate. Analytical HPLC $t_{\mathrm{R}} 10.57 \mathrm{~min}$ (gradient: 5 to $100 \% \mathrm{~B}$ in $20 \mathrm{~min}$ ); $\mathrm{ESI}^{+}-\mathrm{MS}(\mathrm{m} / \mathrm{z}):[\mathrm{M}+\mathrm{H}]^{+}$calcd for $\mathrm{C}_{52} \mathrm{H}_{84} \mathrm{~N}_{21} \mathrm{O}_{17}, 1274.6$; found, 1274.8. After the addition of acetone $(100 \mu \mathrm{L})$ to the crude, the reaction mixture was lyophilized, then resuspended with $t-\mathrm{BuOH} / \mathrm{AcNH}_{4} 100 \mathrm{mM} \mathrm{pH} 7.4(600 \mu \mathrm{L}, 1: 1 v / v)$. Compound $\mathbf{1 b}$ (10 mg, $27 \mu \mathrm{mol}, 4.5$ equiv) and copper micropowder $(600 \mu \mathrm{g}, 10 \mu \mathrm{mol})$ were next added to the solution and the resulting mixture was left under stirring at room temperature. After $4 \mathrm{~h}$, copper was removed by centrifugation and the supernatant purified by semipreparative HPLC. Compound 
8-Fuc/Man was obtained in $88 \%$ yield $(10.0 \mathrm{mg})$. Analytical RP-HPLC: $t_{\mathrm{R}} 7.66 \mathrm{~min}$ (gradient: 5 to $100 \% \mathrm{~B}$ in $20 \mathrm{~min}$ ); ${ }^{1} \mathrm{H}$ NMR $\left(400 \mathrm{MHz}, \mathrm{D}_{2} \mathrm{O}\right) \delta 8.05\left(\mathrm{~s}, 1 \mathrm{H}, \mathrm{H}_{\text {trz }}\right), 8.02(\mathrm{~s}, 2 \mathrm{H}$, $\left.\mathrm{H}_{\mathrm{trz}}\right), 7.79\left(\mathrm{~s}, 1 \mathrm{H}, \mathrm{H}_{\mathrm{ox}}\right), 5.61\left(\mathrm{~d}, J_{1,2}=4.0 \mathrm{~Hz}, 1 \mathrm{H}, \mathrm{H}-1_{\mathrm{Fuc}}\right), 5.60$ (d, $\left.J_{1,2}=4.0 \mathrm{~Hz}, 1 \mathrm{H}, \mathrm{H}-1_{\mathrm{Fuc}}\right), 4.99$ (bs, 3H, 3H-1 Man), 4.85-4.66 (m, 8H, 2 $\left.\mathrm{H}_{\alpha \mathrm{Lys} / \mathrm{Nle}}, 3 \mathrm{CH}_{2 \text { propargyl }}\right), 4.48-4.32(\mathrm{~m}$, $\left.12 \mathrm{H}, 2 \mathrm{H}_{\alpha \mathrm{Lys} / \mathrm{Nle}}, 3 \mathrm{CH}_{2 \varepsilon \mathrm{Nle}}, 2 \mathrm{H}_{\alpha \mathrm{Ala}}, 2 \mathrm{H}_{\alpha \text { Pro }}\right), 4.15-3.64(\mathrm{~m}$, $30 \mathrm{H}, 2 \mathrm{CH}_{2 \alpha \mathrm{Gly}}, 2 \mathrm{CH}_{2 \delta \text { Pro }}$, H-2 $2_{\text {Fuc }}, \mathrm{H}-3_{\text {Fuc }}, \mathrm{H}-4_{\text {Fuc }}, \mathrm{H}-5_{\text {Fuc }}$, $\left.3 \mathrm{H}-2_{\mathrm{Man}}, 3 \mathrm{H}-3_{\mathrm{Man}}, 3 \mathrm{H}-4_{\mathrm{Man}}, 3 \mathrm{H}-5_{\mathrm{Man}}, 3 \mathrm{CH}_{2}-6_{\mathrm{Man}}\right), 3.29-3.19$ (m, 2H, 2H $\left.\mathrm{H}_{\varepsilon \mathrm{Lys}}\right), 2.40-2.30\left(\mathrm{~m}, 2 \mathrm{H}, 2 \mathrm{H}_{\beta \text { Pro }}\right), 2.15-1.28$ (m, $36 \mathrm{H}, 4 \mathrm{CH}_{2 \beta \mathrm{Lys} / \mathrm{Nle}}, 4 \mathrm{CH}_{2 \delta \mathrm{Lys} / \mathrm{Nle}}, 4 \mathrm{CH}_{2 \gamma \mathrm{Lys} / \mathrm{Nle}}, 2 \mathrm{H}_{\beta \text { Pro }}$, $\left.2 \mathrm{CH}_{2 \gamma \text { Pro }}, 2 \mathrm{CH}_{3 \mathrm{Ala}}\right), 1.22\left(\mathrm{~d}, J_{5,6}=6.6 \mathrm{~Hz}, 3 \mathrm{H}, \mathrm{CH}_{3 \mathrm{Fuc}}\right)$ $\mathrm{ESI}^{+}-\mathrm{MS}(\mathrm{m} / \mathrm{z}):[\mathrm{M}+\mathrm{H}]^{+}$calcd for $\mathrm{C}_{79} \mathrm{H}_{126} \mathrm{~N}_{21} \mathrm{O}_{34}, 1912.9$; found, 1913.2. Compounds 8-Man/Fuc, 8-Man/Lac, 8-Fuc/ Lac, 8-Lac/Man and 8-Lac/Fuc were prepared under the same experimental conditions.

\section{Supporting Information}

The Supporting Information file contains analytical details of all heteroglycoclusters of series 2:2 (6-Man/Fuc,

6-Man/Lac, 6-Fuc/Man, 6-Fuc/Lac, 6-Lac/Man and 6-Lac/Fuc) and 3:1 (8-Man/Fuc, 8-Man/Lac, 8-Fuc/Man, 8-Fuc/Lac, 8-Lac/Man and 8-Lac/Fuc) described in this article.

\section{Supporting Information File 1}

Crude RP-HPLC profiles and ESI-MS spectra for the heteroglycoclusters.

[http://www.beilstein-journals.org/bjoc/content/ supplementary/1860-5397-8-47-S1.pdf]

\section{Acknowledgements}

This work was supported by the Université Joseph Fourier (UJF) and the Centre National de la Recherche Scientifique (CNRS). We are grateful to the "Communauté d'agglomération Grenoble-Alpes Métropole" (Nanobio program) for access to the facilities of the Synthesis platform. Financial support from the "Cluster de Recherche Chimie de la Région Rhône-Alpes" (B.T.) and from the pôle Chimie, Sciences du vivant et de la santé, Bioingénierie of UJF (M. F.) are duly acknowledged.

\section{References}

1. Varki, A. Glycobiology 1993, 3, 97-130. doi:10.1093/glycob/3.2.97

2. Chabre, Y. M.; Roy, R. Adv. Carbohydr. Chem. Biochem. 2010, 63, 165-393. doi:10.1016/S0065-2318(10)63006-5

3. Imberty, A.; Chabre, Y. M.; Roy, R. Chem.-Eur. J. 2008, 14, 7490-7499. doi:10.1002/chem.200800700

4. Pieters, R. J. Org. Biomol. Chem. 2009, 7, 2013-2025. doi:10.1039/b901828j
5. Renaudet, O.; Spinelli, N., Eds. Synthesis and biological applications of glycoconjugates; Bentham Science Publishers Ltd.: U.A.E., 2011.

6. Lee, Y. C.; Lee, R. T. Acc. Chem. Res. 1995, 28, 321-327. doi:10.1021/ar00056a001

7. Lundquist, J. J.; Toone, E. J. Chem. Rev. 2002, 102, 555-578. doi:10.1021/cr000418f

8. Kitov, P. I.; Sadowska, J. M.; Mulvey, G.; Armstrong, G. D.; Ling, H.; Pannu, N. S.; Read, R. J.; Bundle, D. R. Nature 2000, 403, 669-672. doi:10.1038/35001095

9. Touaibia, M.; Wellens, A.; Shiao, T. C.; Wang, Q.; Sirois, S.; Bouckaert, J.; Roy, R. ChemMedChem 2007, 2, 1190-1201. doi:10.1002/cmdc.200700063

10. Joosten, J. A. F.; Loimaranta, V.; Appeldoorn, C. C. M.; Haataja, S.; El Maate, F. A.; Liskamp, R. M. J.; Finne, J.; Pieters, R. J. J. Med. Chem. 2004, 47, 6499-6508. doi:10.1021/jm049476+

11. Horan, N.; Yan, L.; Isobe, H.; Whitesides, G. M.; Kahne, D. Proc. Natl. Acad. Sci. U. S. A. 1999, 96, 11782-11786. doi:10.1073/pnas.96.21.11782

12. Gómez-García, M.; Benito, J. M.; Rodríguez-Lucena, D.; Yu, J.-X.; Chmurski, K.; Ortiz Mellet, C.; Gutiérrez Gallego, R.; Maestre, A.; Defaye, J.; Garcia Fernández, J. M. J. Am. Chem. Soc. 2005, 127, 7970-7971. doi:10.1021/ja050934t

13. Ramström, O.; Lehn, J.-M. ChemBioChem 2000, 1, 41-48. doi:10.1002/1439-7633(20000703)1:1<41::AID-CBIC41>3.0.CO;2-L

14. Ramström, O.; Lohmann, S.; Bunyapaiboonsri, T.; Lehn, J.-M. Chem.-Eur. J. 2004, 10, 1711-1715. doi:10.1002/chem.200305551

15. Gómez-García, M.; Benito, J. M.; Gutiérrez-Gallego, R.; Maestre, A.; Ortiz Mellet, C.; García Fernández, J. M.; Jiménez Blanco, J. L. Org. Biomol. Chem. 2010, 8, 1849-1860. doi:10.1039/b920048g

16. Ortega-Muñoz, M.; Perez-Balderas, F.; Morales-Sanfrutos, J.; Hernandez-Mateo, F.; Isac-García, J.; Santoyo-Gonzalez, F. Eur. J. Org. Chem. 2009, 2454-2473. doi:10.1002/ejoc.200801169 17. Deguise, I.; Lagnoux, D.; Roy, R. New J. Chem. 2007, 31, 1321-1331. 18. Katajisto, J.; Karskela, T.; Heinonen, P.; Lönnberg, H. J. Org. Chem. 2002, 67, 7995-8001. doi:10.1021/jo026053b

19. Patel, A.; Lindhorst, T. K. Eur. J. Org. Chem. 2002, 79-86. doi:10.1002/1099-0690(20021)2002:1<79::AID-EJOC79>3.0.CO;2-1

20. Geng, J.; Mantovani, G.; Tao, L.; Nicolas, J.; Chen, G.; Wallis, R.; Mitchell, D. A.; Johnson, B. R. G.; Evans, S. D.; Haddleton, D. M. J. Am. Chem. Soc. 2007, 129, 15156-15163. doi:10.1021/ja072999x

21. Ladmiral, V.; Mantovani, G.; Clarkson, G. J.; Cauet, S.; Irwin, J. L.; Haddleton, D. M. J. Am. Chem. Soc. 2006, 128, 4823-4830. doi:10.1021/ja058364k

22. Fiore, M.; Chambery, A.; Marra, A.; Dondoni, A. Org. Biomol. Chem. 2009, 7, 3910-3913. doi:10.1039/b912686d

23. Lindhorst, T. K.; Bruegge, K.; Fuchs, A.; Sperling, O. Beilstein J. Org. Chem. 2010, 6, 801-809. doi:10.3762/bjoc.6.90

24. Liang, C.-H.; Wang, S.-K.; Lin, C.-W.; Wang, C.-C.; Wong, C.-H.; Wu, C.-Y. Angew. Chem., Int. Ed. 2011, 50, 1608-1612. doi:10.1002/anie.201003482

25. Gómez-García, M.; Benito, J. M.; Butera, A. P.; Ortiz Mellet, C.; García Fernández, J. M.; Jiménez Blanco, J. L. J. Org. Chem. 2012, 77, 1273-1288. doi:10.1021/jo201797b

26. Duléry, V.; Renaudet, O.; Wilczewski, M.; Van der Heyden, A.; Labbé, P.; Dumy, P. J. Comb. Chem. 2008, 10, 368-371. doi:10.1021/cc800029v

27. Renaudet, O.; BenMohamed, L.; Dasgupta, G.; Bettahi, I.; Dumy, P. ChemMedChem 2008, 3, 737-741. doi:10.1002/cmdc.200700315 
28. Renaudet, O.; Dasgupta, G.; Bettahi, I.; Shi, A.; Nesburn, A. B.; Dumy, P.; BenMohamed, L. PLoS One 2010, 5, e11216. doi:10.1371/journal.pone.0011216

29. Renaudet, O.; Křenek, K.; Bossu, I.; Dumy, P.; Kádek, A.; Adámek, D.; Vaněk, O.; Kavan, D.; Gažák, R.; Šulc, M.; Bezouška, K.; Křen, V. J. Am. Chem. Soc. 2010, 132, 6800-6808. doi:10.1021/ja101296t

30. Bossu, I.; Šulc, M.; Křenek, K.; Dufour, E.; Garcia, J.; Berthet, N.; Dumy, P.; Křen, V.; Renaudet, O. Org. Biomol. Chem. 2011, 9, 1948-1959. doi:10.1039/c0ob00772b

31. Pujol, A. M.; Cuillel, M.; Renaudet, O.; Lebrun, C.; Charbonnier, P.; Cassio, D.; Gateau, C.; Dumy, P.; Mintz, E.; Delangle, P. J. Am. Chem. Soc. 2011, 133, 286-296. doi:10.1021/ja106206z

32. Tornøe, C. W.; Christensen, C.; Meldal, M. J. Org. Chem. 2002, 67, 3057-3064. doi:10.1021/jo011148j

33. Rostovtsev, V. V.; Green, L. G.; Fokin, V. V.; Sharpless, K. B. Angew. Chem., Int. Ed. 2002, 41, 2596-2599. doi:10.1002/1521-3773(20020715)41:14<2596::AID-ANIE2596>3.0.CO ;2-4

34. Dondoni, A. Chem.-Asian J. 2007, 2, 700-708 doi:10.1002/asia.200700015

35. Jewett, J. C.; Bertozzi, C. R. Chem. Soc. Rev. 2010, 39, 1272-1279. doi:10.1039/b901970g

36. Galibert, M.; Renaudet, O.; Dumy, P.; Boturyn, D. Angew. Chem., Int. Ed. 2011, 50, 1901-1904. doi:10.1002/anie.201006867

37. Clavé, G.; Volland, H.; Flaender, M.; Gasparutto, D.; Romieu, A.; Renard, P.-Y. Org. Biomol. Chem. 2010, 8, 4329-4345. doi:10.1039/c0ob00133c

38. Cao, S.; Tropper, F. D.; Roy, R. Tetrahedron 1995, 51, 6679-6686. doi:10.1016/0040-4020(95)00325-3

39. Renaudet, O.; Dumy, P. Org. Biomol. Chem. 2006, 4, 2628-2636. doi:10.1039/b604391g

40. Duléry, V.; Renaudet, O.; Philouze, C.; Dumy, P. Carbohydr. Res. 2007, 342, 894-900. doi:10.1016/j.carres.2007.02.003

41. Fernandez-Megia, E.; Correa, J.; Rodriguez-Meizoso, I.; Riguera, R. Macromolecules 2006, 39, 2113-2120. doi:10.1021/ma052448w

42. Bossu, I.; Berthet, N.; Dumy, P.; Renaudet, O. J. Carbohydr. Chem. 2011, 30, 458-468. doi:10.1080/07328303.2011.590260

\section{License and Terms}

This is an Open Access article under the terms of the Creative Commons Attribution License (http://creativecommons.org/licenses/by/2.0), which permits unrestricted use, distribution, and reproduction in any medium, provided the original work is properly cited.

The license is subject to the Beilstein Journal of Organic Chemistry terms and conditions: (http://www.beilstein-journals.org/bjoc)

The definitive version of this article is the electronic one which can be found at: doi:10.3762/bjoc. 8.47 